\title{
Intraoperative renal near-infrared spectroscopy indicates developing acute kidney injury in infants undergoing cardiac surgery with cardiopulmonary bypass: a case-control study
}

Bettina Ruf ${ }^{1 *}$, Vittorio Bonelli², Gunter Balling ${ }^{1}$, Jürgen Hörer ${ }^{3}$, Nicole Nagdyman', Siegmund Lorenz Braun ${ }^{4}$, Peter Ewert ${ }^{1}$ and Karl Reiter ${ }^{5}$

\begin{abstract}
Introduction: Acute kidney injury (AKI) is a frequent complication after cardiac surgery with cardiopulmonary bypass in infants. Renal near-infrared spectroscopy (NIRS) is used to evaluate regional oximetry in a non-invasive continuous real-time fashion, and reflects tissue perfusion. The aim of this study was to evaluate the relationship between renal oximetry and development of AKI in the operative and post-operative setting in infants undergoing cardiopulmonary bypass surgery.

Methods: In this prospective study, we enrolled 59 infants undergoing cardiopulmonary bypass surgery for congenital heart disease for univentricular $(n=26)$ or biventricular $(n=33)$ repair. Renal NIRS was continuously measured intraoperatively and for at least 24 hours postoperatively and analysed for the intraoperative and first 12 hours, first 24 hours and first 48 hours postoperatively. The renal oximetry values were correlated with the paediatric risk, injury, failure, loss, end (pRIFLE) classification for AKI, renal biomarkers and the postoperative course.

Results: Twenty-eight (48\%) infants developed AKI based on pRIFLE classification. Already during intraoperative renal oximetry and further in the first 12 hours, 24 hours and 48 hours postoperatively, significantly lower renal oximetry values in AKI patients compared with patients with normal renal function were recorded $(P<0.05)$. Of the 28 patients who developed AKI, 3 (11\%) needed renal replacement therapy and 2 (7\%) died. In the non-AKI group, no deaths occurred. Infants with decreased renal oximetry values developed significantly higher lactate levels 24 hours after surgery. Cystatin C was a late parameter of AKI, and neutrophil gelatinase-associated lipocalin values were not correlated with AKI occurrence.

Conclusion: Our results suggest that prolonged low renal oximetry values during cardiac surgery correlate with the development of AKI and may be superior to conventional biochemical markers. Renal NIRS might be a promising non-invasive tool of multimodal monitoring of kidney function and developing AKI in infants undergoing cardiac surgery with cardiopulmonary bypass.
\end{abstract}

\footnotetext{
*Correspondence: ruf@dhm.mhn.de

'Department of Pediatric Cardiology and Congenital Heart Disease, German Heart Centre Munich, Technical University, Lazarettstr. 36, 80636 Munich,

Germany

Full list of author information is available at the end of the article
} 


\section{Introduction}

Acute kidney injury (AKI) is a frequent complication after cardiac surgery with cardiopulmonary bypass (CPB) in infants. In a recent multicentre study including 311 paediatric patients after on-pump cardiac surgery, $42 \%$ developed AKI postoperatively [1]. Especially patients younger than 2 years showed an increased risk for AKI [1]. AKI after СРB surgery is associated with longer time on mechanical ventilation and intensive care unit (ICU) stay [1-5] and possibly increased mortality [3,4].

Criteria for the definition of paediatric AKI have been defined by the paediatric modification of the risk, injury, failure, loss, end (pRIFLE) classification. More recently, these criteria were refined by the Acute Kidney Injury Network, but basically they rely on increases in serum creatinine or decreases in creatinine clearance [6]. These parameters reach their maximum in most $(78 \%)$ of the cases only after 48 hours [1]. Therefore, much effort has been spent on the identification of reliable biomarkers for early detection of AKI (for example, neutrophil gelatinase-associated lipocalin (NGAL), interleukin-18, kidney injury molecule-1 (KIM-1) [7]); however, predictive values vary considerably between studies, and no single substance has been shown to have consistent sensitivity [8]. Most promising in this regard is NGAL, for which, in a recent study in paediatric post-cardiac surgery patients, area under the receiver operating characteristic curve (AUROC) values ranging from 0.68 to 0.92 within the first 15 hours after surgery were found [9].

Near-infrared spectroscopy (NIRS) is a non-invasive, continuous method of evaluating real-time regional oximetry $\left(\mathrm{rSO}_{2}\right)$. The technology is based on the different absorption of near-infrared wavelengths by oxygenated and deoxygenated haemoglobin. The transmitter and the receiving optodes are placed ipsilateral in the sensor. This design capitalizes on the fact that photons transmitted through a sphere will traverse an elliptical path in which the mean depth of penetration is proportional to the transmitter and receiver optode separation [10].

Several studies have shown a moderate correlation between cerebral and somatic NIRS monitoring and markers of cardiac output such as lactate or systemic venous oxygen saturation $\left(\mathrm{ScrO}_{2}\right)$ and regional tissue perfusion in a variety of clinical settings [10-13]. However, NIRS measurements in different organs have shown variable correlations to systemic oxygenation parameters $[11,14]$ or no reliable correlation at all [15].

In a recent study, a correlation was demonstrated between urinary biomarkers and low renal NIRS saturations and a compound outcome, including the need for renal replacement therapy in infants after cardiac surgery [16]. Importantly, no correlation with AKI occurrence was noted.

In our present study, we hypothesized that early and extended continuous renal NIRS recordings starting intraoperatively and continued for at least 24 hours postoperatively would correlate with renal injury measured by biomarkers and pRIFLE stage. We applied a modified renal NIRS threshold to account for low saturations in infants with cyanotic heart lesions and calculated a modified NIRS score that included magnitude and duration of renal desaturations.

\section{Methods}

\section{Patients and study design}

In this prospective study, we consecutively enrolled infants under 12 months of age who underwent cardiac surgery on $\mathrm{CPB}$ after written informed consent was obtained from their parents. Infants with preexisting renal disease, sepsis or multiple organ dysfunction were excluded. Ethical approval for the study protocol was given by the research ethics committee at German Heart Centre Munich, Technical University of Munich, Germany.

\section{Near-infrared spectroscopy}

We measured cerebral and renal oximetry with the INVOS monitor (Covidien/Somanetics, Dublin, Ireland) intraoperatively and 24 to 48 hours postoperatively. The NIRS sensor was placed after intubation before starting the operation on the left or right forehead for cerebral oximetry and on the back left or right of the spine at the level of T10-L2 for renal oximetry. Because of the importance of a small skin organ distance, we included only infants younger than 12 month of age and below $10 \mathrm{~kg}$ body weight. The $\mathrm{rSO}_{2}$ was recorded continuously every 2 seconds after intubation, during $\mathrm{CPB}$ and until the end of the surgical procedure. These values represent the intraoperative NIRS measurements. After admission to the ICU, the renal and cerebral oximetry was restarted and recorded continuously for at least 24 hours in each patient. Renal oximetry measurements were continued until extubation or for a maximum of 48 hours postoperatively. The measurements over the first 24 hours postoperatively were calculated for the first 12 hours and the whole first 24-hour period. Values obtained during the 24 hours following the first 24-hour period were analysed separately. In this study, we focused on renal oximetry; therefore, cerebral NIRS values are not presented.

According to our knowledge, validated critical baselines or thresholds for renal $\mathrm{rSO}_{2}$ are lacking and do not discern between cyanotic and acyanotic cardiac lesions. We decided to set two different thresholds. According to the critical baseline of $45 \%$ to $55 \%$ in cerebral NIRS studies [17-19] and the 10 to 20 points higher somatic than cerebral values in healthy infants $[20,21]$, we set the critical baselines at $65 \% \mathrm{rSO}_{2}$ for renal NIRS. To account for a priori lower NIRS values in patients with cyanotic lesions, we defined a second criterion, which includes relative decreases in regional oxygenation compared with 
an individually calculated baseline. In each patient, following stabilization after intubation, renal $\mathrm{rSO}_{2}$ was measured over 10 minutes and values were averaged to define a baseline $\mathrm{rSO}_{2}$ value. The threshold was defined as a $25 \%$ $\mathrm{rSO}_{2}$ decrease compared with the baseline value.

After recording the renal oximetry values intra- and postoperatively, a renal $\mathrm{rSO}_{2}$ score was calculated. This score has been established in studies on cerebral NIRS after cardiac surgery in adult patients, but up to now not in infants [17]. It represents an area under the curve (AUC) measurement that reflects both the depth and duration of desaturations below the set thresholds (Figures 1 and 2). We adapted the score for long-term measurements by using minutes instead of seconds in the numerator, which gives the formula: $\mathrm{rSO}_{2}$ score $=$ (baseline $\mathrm{rSO}_{2}$ - current $\left.\mathrm{rSO}_{2}(\%)\right) \times$ time (minutes). In this article, the following abbreviation for the two renal NIRS thresholds are used: rNIRS65 = renal $\mathrm{rSO}_{2}<65 \%$ and rNIRS25 $=$ renal $\mathrm{rSO}_{2}$ decrease of $25 \%$ compared with a preoperative baseline value.

\section{Laboratory parameters}

Serum creatinine, urea and cystatin $\mathrm{C}$ as well as urine volume and urinary creatinine were measured preoperatively, within 1 hour postoperatively and on days 1 to 5 after the surgical procedure.

Creatinine levels in plasma and urine were determined using a kinetic colorimetric assay based on the Jaffé method. For the determination of urea/urea nitrogen in plasma, a kinetic test with urease and glutamate dehydrogenase. For cystatin $\mathrm{C}$ measurement, a latex particle-enhanced immunoturbidimetric assay was used. All three tests were done with the Cobas Integra 800 analyser (Roche Diagnostics, Mannheim, Germany).

Urinary NGAL concentrations were measured by performing a solid-phase, enzyme-linked immunosorbent assay based on the sandwich principle (Hycult Biotech, Cambridge, UK). The determination of NGAL in plasma anticoagulated with ethylenediaminetetraacetic acid was performed with a fluorescence immunoassay using the Triage MeterPro (Alere, Cologne, Germany).

\section{Perioperative course}

The following clinical parameters were recorded: operation time, time on bypass, aortic cross-clamp time, circulatory arrest time, time on a respirator, length of stay in the ICU and until hospital discharge. As indirect markers of cardiac output lactate at 6 hours, 12 hours and 24 hours postoperatively, $\mathrm{ScvO}_{2}$ and arterial oxygen saturation 2 hours and 24 hours postoperatively were collected. Daily urine output related to body weight and need of renal replacement therapy (peritoneal dialysis or continuous venovenous haemodiafiltration) were noted. The Risk Adjustment for Congenital Heart Surgery (RACHS-1) was used to categorize and compare the severity of the performed cardiac surgery. This instrument gives a score to stratify anatomic diversity into six categories based on type of performed surgery, age at operation and similar in-hospital mortality [22].

According to the pRIFLE classification [6], AKI was defined as a $50 \%$ or more increase of serum creatinine. Creatinine was routinely measured preoperatively and in

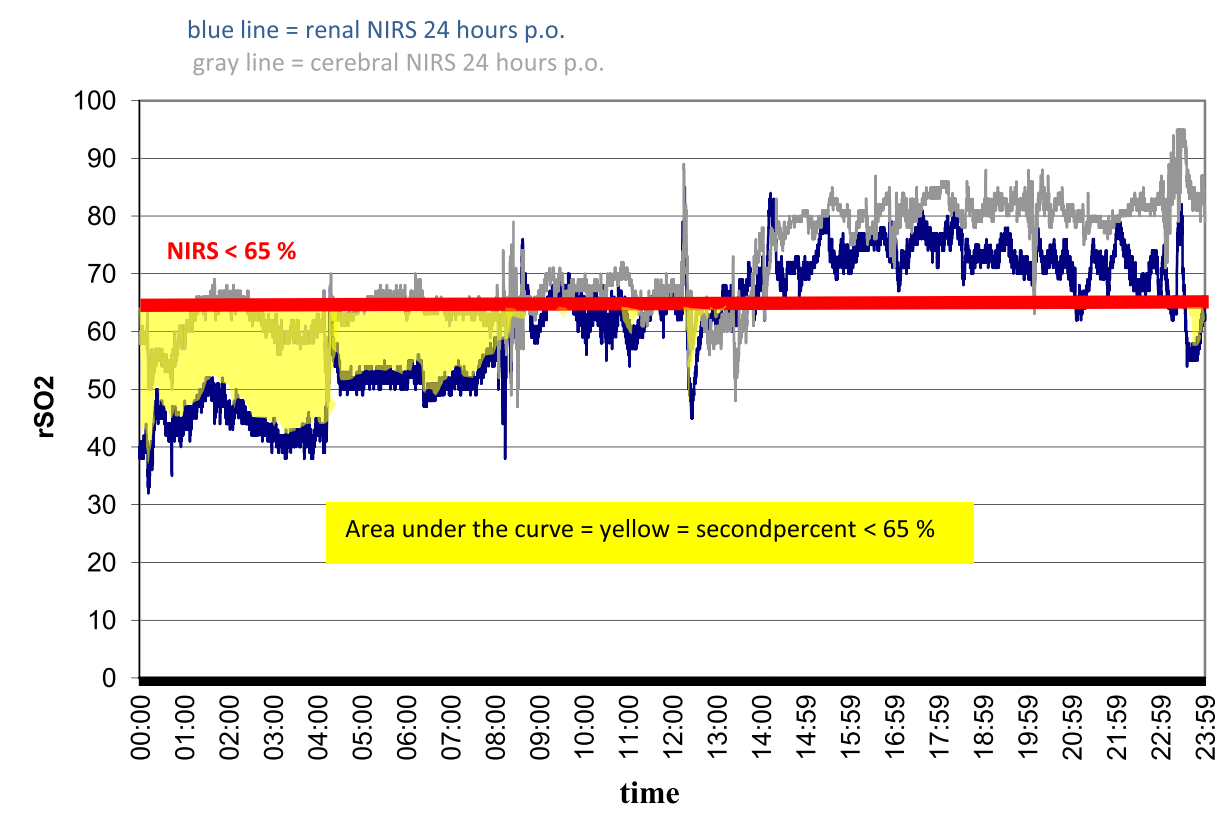

Figure 1 Example of calculating the area under the curve for regional oxygenation below $65 \%$. Yellow region represents the second percent with the renal near-infrared spectroscopy (NIRS) below the $65 \%$ threshold (red bar). p.o., Postoperatively; $\mathrm{rSO}_{2}$, Regional oxygenation. 


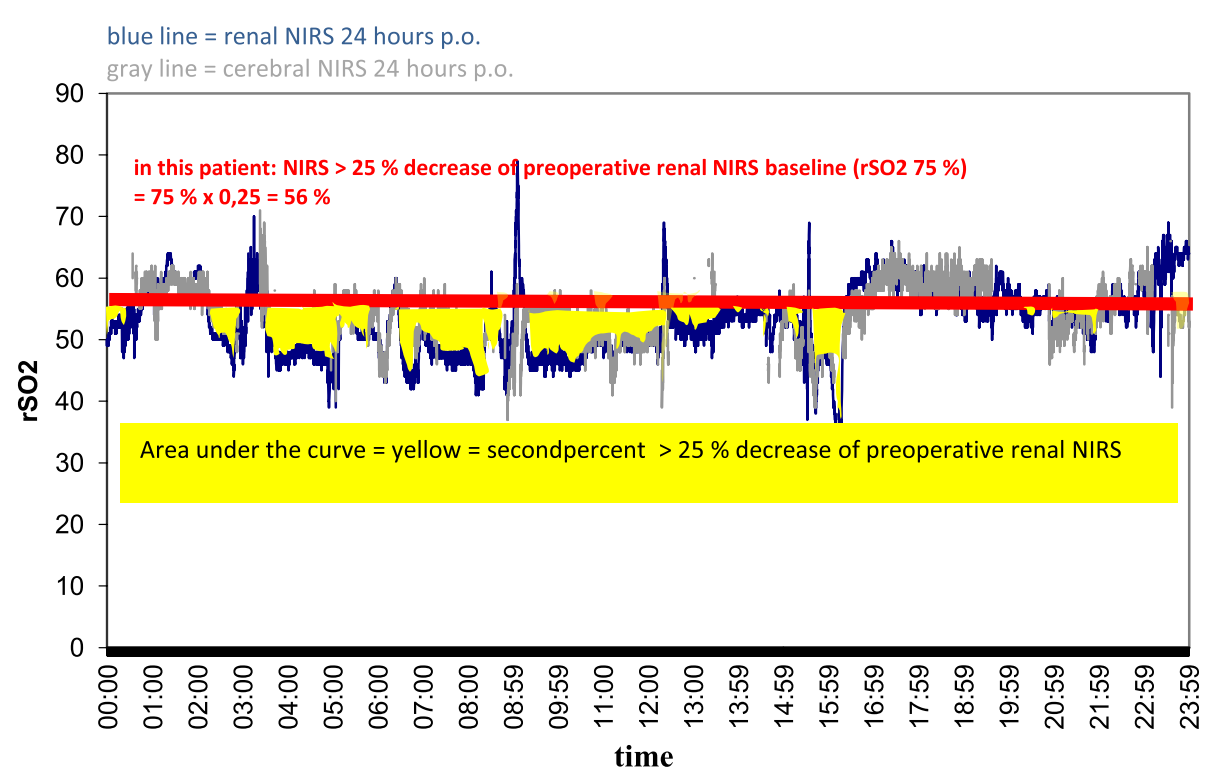

Figure 2 Example of calculating the area under the curve with the baseline of $>\mathbf{2 5} \%$ decrease of the preoperative individual averaged value of renal near-infrared spectroscopy. In this case, the area is calculated as $75 \% \times 0.25=56 \%$. NIRS, Near-infrared spectroscopy; rSO $_{2}$, Regional oxygenation.

the morning following surgery. Urine volume criteria were not used, owing to variable diuretic use.

\section{Statistical analysis}

We used the $t$-test for independent samples for symmetric data distribution, and we performed a two-sided $x^{2}$ test for categorical variables and a two-sided Mann-Whitney $U$ test for continuous variables in case of an asymmetric data distribution. Data are presented as median and range or as individual values. Correlation analysis was performed with Spearman's $\rho$ coefficient of correlation for asymmetric data distribution. ROC analysis was used for calculating sensitivity, specificity and positive and negative predictive values. Results were considered statistically significant at $P<0.05$. For power analysis, we assumed postoperative AKI to occur in $30 \%$ of patients. If a clear cutoff value in the NIRS measurements was obtained, we calculated, using standard tables, a sample size of 56 patients to reach a power of $80 \%$ and a $P$-value $<0.05$. The statistical calculations were done using SPSS 21 software (IBM SPSS, Chicago, IL, USA).

\section{Results}

From January 2011 to August 2011, we enrolled 59 infants undergoing $\mathrm{CPB}$ cardiac surgery because of congenital heart defects. According to the pRIFLE classification scheme [6], we determined that $48 \%$ (28 of 59) of the infants developed AKI after CPB. A creatinine increase by $50 \%$ of the preoperative baseline value was first seen in the serum creatinine at 24 hours postoperatively; hence, AKI was diagnosed at this time point or later in our cohort. Figure 3 demonstrates the course of serum creatinine levels in infants with versus without AKI.

The infants who developed AKI were significantly younger than the infants who did not. For the demographic data of the two groups, see Table 1.

There was no significant difference between the two groups in operation time, bypass time, aortic cross clamp and circulatory arrest time. The infants who developed AKI were significantly more often palliated with an aortopulmonary shunt than infants without AKI. The RACHS-1 was not significantly different between the two groups. The surgical data and RACHS-1 scores are listed in Table 2.

Plasma lactate was significantly higher and $\mathrm{ScvO}_{2}$ significantly lower at 24 hours postoperatively in the AKI group versus the non-AKI group (Table 3). Three (11\%) of twenty-eight infants with AKI needed renal replacement therapy (one peritoneal dialysis, two continuous venovenous haemodiafiltration) after resuscitation at the end of surgery (one case) and on postoperative days 3 and 4 (two cases). Two (7\%) of twenty-eight infants with AKI died. There was no fatal course in the non-AKI group. Time on mechanical ventilation was significantly longer in infants with AKI compared with infants without AKI. Table 3 presents the clinical data of the postoperative course.

The renal $\mathrm{rSO}_{2}$ during the intraoperative course showed significantly higher $\mathrm{rSO}_{2}$ scores (and therefore worse oxygenation values) in infants with AKI than in infants without AKI for both baseline definitions. Infants with AKI demonstrated a significantly higher median 

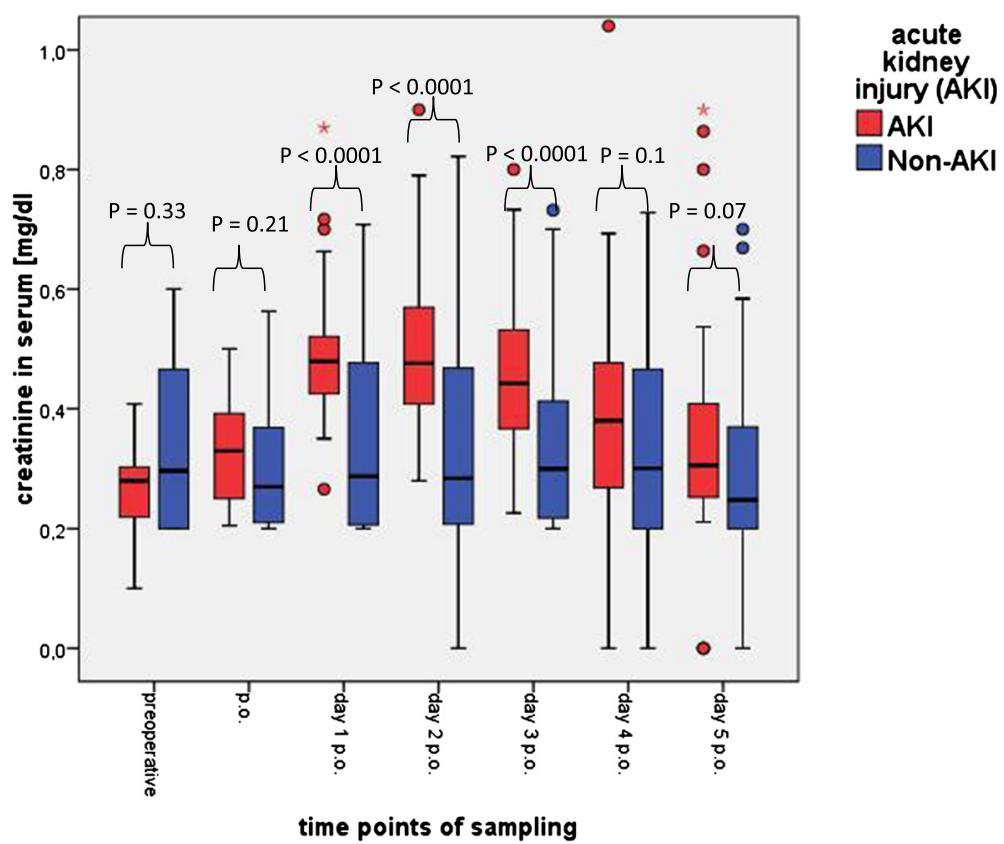

Figure 3 The pre- and postoperative course of serum creatinine in infants with versus without acute kidney injury who underwent cardiopulmonary bypass surgery is presented as a boxplot. AKI, Acute kidney injury; p.o., Postoperatively.

rNIRS65 score (598 min\%; range: 86 to 2,$532 ; P=0.01$ ) compared with infants without AKI (154 min\%; range: 0 to 2,$997 ; P=0.001)$. The median rNIRS25 score was also significantly higher in infants with AKI (131 min\%; range: 0 to 1,200$)$ than in infants without AKI ( 0 min\%; range: 0 to 2,263; $P=0.001$ ) (Table 4, Figures 4 and 5). Postoperatively, both renal $\mathrm{rSO}_{2}$ scores were significantly higher 12 and 24 hours after surgery in the AKI versus the non-AKI group (rNIRS65 score at 12 hours: 694 (0 to 11,534) $\mathrm{min} \%$ in the AKI vs. 26 (0 to 3,124$) \mathrm{min} \%$ in the non-AKI group $(P=0.02)$; rNIRS65 at 24 hours: 3,747 (0 to $22,590) \mathrm{min} \%$ vs. 267 (1 to 9,417) $\mathrm{min} \%$ in the non-AKI group; $P=0.007$ ) (Table 4 , Figures 4 and 5). At 12 hours, infants with AKI showed a rNIRS25 score of 4 (0 to 7,091) min\% compared with 0 (0 to 1,772$) \min \%$ in infants without AKI $(P=0.04)$, and at 24 hours, rNIRS25 scores were 214 ( 0 to 13,267$)$ min\% compared with 4 ( 0 to 312 ) min\% in the AKI versus non-AKI group, respectively $(P=0.02)$ (Table 4 , Figures 4 and 5$)$. Forty-eight hours postoperatively, only the rNIRS65 score was significantly higher in the AKI in comparison with the non-AKI group.

Renal NIRS values of patients with cyanosis compared with patients without cyanosis independent of AKI showed no significant difference at the given time points. There was a moderate negative correlation between the arterial oxygen saturation 2 hours postoperatively with the rNIRS65 score within 12 hours $(r=0.5 ; P=0.01)$ and 24 hours $(r=0.4 ; P=0.07)$ after surgery, but not the postoperative rNIRS25 score.

We found a significant moderate correlation between the intraoperative rNIRS65 and intraoperative rNIRS25 scores and the arterial lactate 6 hours postoperatively ( $r=0.4, P<0.0001$, and $r=0.4, P=002$; respectively). The rNIRS65 and rNIRS25 scores in the first 24 hours

Table 1 Demographic data of infants with versus without acute kidney injury after undergoing cardiopulmonary bypass surgery ${ }^{a}$

\begin{tabular}{llll}
\hline & AKI & No AKI & P-value \\
\hline Age at operation (days) & $27(5$ to 274$)$ & $105(8$ to 305$)$ & 0.03 \\
Weight at operation $(\mathrm{g})$ & $3,355(2,445$ to 9,100$)$ & $4,490(2,260$ to 7,530$)$ & 0.1 \\
Height at operation $(\mathrm{cm})$ & $52(44$ to 75$)$ & $59(41$ to 71$)$ & 0.03 \\
Female, $n(\%)$ & $10(36)$ & $14(45)$ & 0.8 \\
Male, $n(\%)$ & $18(64)$ & $17(55)$ & $0.26(0.15$ to 0.36$)$ \\
BSA at operation $\left(\mathrm{m}^{2}\right)$ & $0.22(0.17$ to 0.41$)$ & 0.06 \\
\hline
\end{tabular}

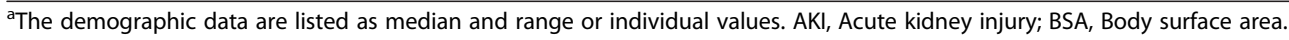


Table 2 Surgery-related data and Risk Adjustment for Congenital Heart Surgery scores of infants with versus without acute kidney injury after undergoing cardiopulmonary bypass surgery ${ }^{a}$

\begin{tabular}{|c|c|c|c|}
\hline & AKI & No AKI & $P$-value \\
\hline Biventricular correction, $n(\%)$ & $11(39 \%)$ & $22(71 \%)$ & 0.01 \\
\hline Univentricular palliation: cyanotic, $n$ (\%) & $17(61 \%)$ & $9(29 \%)$ & 0.01 \\
\hline Shunt, $n(\%)$ & $15(54 \%)$ & $3(10 \%)$ & $P<0.01$ \\
\hline PCPC, $n(\%)$ & $2(7 \%)$ & $6(19 \%)$ & \\
\hline Operation time (min) & $174(80$ to 530$)$ & 150 (80 to 555$)$ & 0.45 \\
\hline Bypass (min) & 75 (30 to 150$)$ & 77 (23 to 160$)$ & 0.9 \\
\hline Aortic cross-clamp (min) & 28 (0 to 83 ) & 47 (0 to 110$)$ & 0.07 \\
\hline Circulatory arrest (min) & $0(0$ to 53$)$ & 0 (0 to 44) & 0.12 \\
\hline \multicolumn{4}{|l|}{ RACHS-1 score } \\
\hline 2 and $3, n(\%)$ & $15(56)$ & $22(69)$ & 0.42 \\
\hline 4 to $6, n(\%)$ & $12(44)$ & $10(31)$ & 0.42 \\
\hline
\end{tabular}

${ }^{a}$ The operation data and the Risk Adjustment for Congenital Heart Surgery (RACHS-1) score are listed as median and range or individual values. AKI, Acute kidney injury; PCPC, Partial cavopulmonary connection.

postoperatively showed a moderate correlation to the $\mathrm{ScvO}_{2}$ at 2 and 24 hours after surgery (rNIRS65 score to $\mathrm{ScvO}_{2}$ at 2 hours: $r=0.6, P<0.0001$; rNIRS65 score to $\mathrm{ScvO}_{2}$ at 24 hours: $r=0.5, P<0.0001$; rNIRS25 score to $\mathrm{ScvO}_{2}$ at 2 hours: $r=0.5, P=0.001$; rNIRS25 score to $\mathrm{ScvO}_{2}$ at 24 hours: $r=0.4, P=0.009$ ).

In the logistic regression analysis, the only significant parameter for AKI development was the blood pressure in the first 24 hours postoperatively $\left(R^{2}=0.3, P=0.02\right)$. No significance was found for age, cyanosis, bypass time or $\mathrm{ScvO}_{2}$ at 2 hours postoperatively.
The ROC analysis showed that, for an intraoperative rNIRS65 score cutoff value of $95 \mathrm{~min} \%$, the sensitivity was $96 \%$ and specificity was $40 \%$ (AUC, $0.62 ; P=0.12$ ) in capturing postoperative AKI. The positive predictive value (PPV) was $59 \%$, and the negative predictive value (NPV) was $88 \%$. For an intraoperative cutoff value for the rNIRS25 score of $23 \mathrm{~min} \%$, we found a sensitivity of $67 \%$ and a specificity of $76 \%$ (AUC, $0.69 ; P=0.01$ ). The PPV was $71 \%$, and the NPV was $76 \%$.

Cystatin $\mathrm{C}$ showed a significant increase after $\mathrm{CPB}$ surgery in patients with AKI starting at 24 hours

Table 3 Postoperative clinical data of infants with versus without acute kidney injury after undergoing cardiopulmonary bypass surgery ${ }^{a}$

\begin{tabular}{|c|c|c|c|}
\hline & AKI & No AKI & $P$-value \\
\hline Lactate 6 hr p.o. ( $\mu \mathrm{mol} / \mathrm{L})$ & $2.2(0.9$ to 17$)$ & $1.9(0.9$ to 7.4$)$ & 0.32 \\
\hline Lactate 12 hr p.o. ( $\mu \mathrm{mol} / \mathrm{L})$ & $3(1.1$ to 19$)$ & 2.4 (1 to 6$)$ & 0.08 \\
\hline Lactate 24 hr p.o. ( $\mu \mathrm{mol} / \mathrm{L})$ & $2.6(1.4$ to 16$)$ & $1.7(0.9$ to 6.5$)$ & 0.01 \\
\hline $\mathrm{ScvO}_{2} 2$ hr p.o. (\%) & 57 (35 to 88) & 60 (34 to 87) & 0.54 \\
\hline $\mathrm{ScvO}_{2} 24$ hr p.o. (\%) & 55 (40 to 88$)$ & $62(44$ to 89$)$ & 0.004 \\
\hline $\mathrm{SaO}_{2} 2$ hr p.o. (\%) & 87 (70 to 100$)$ & $92(55$ to 99$)$ & 0.39 \\
\hline $\mathrm{SaO}_{2} 24$ hr p.o. (\%) & $83(69$ to 100$)$ & 93 (63 to 100) & 0.76 \\
\hline MAP $<50$ mmHg $>2$ hr within 24 hr p.o., $n$ (\%) & $16(59)$ & $8(25)$ & 0.008 \\
\hline Norepinephrine $\leq 0.1$ Mg/kg/min, n (\%) & $15(56)$ & $11(34)$ & 0.03 \\
\hline Norepinephrine >0.1 mg/kg/min, $n$ (\%) & $5(19)$ & $2(6)$ & 0.01 \\
\hline Urine output 24 hr p.o. (ml/kg) & 4.9 (1.5 to 7.9$)$ & $4.6(2.9$ to 8.8$)$ & 0.37 \\
\hline Time on respirator (hr) & 47 (2 to 624) & 22 (2 to 270$)$ & 0.03 \\
\hline ICU stay (days) & 12 (3 to 35$)$ & 10 (4 to 74$)$ & 0.06 \\
\hline Hospital stay (days) & $28(7$ to 76$)$ & 25 (12 to 96$)$ & 0.13 \\
\hline RRT, $n(\%)$ & $3(11)$ & $0(0)$ & 0.09 \\
\hline Deaths, $n(\%)$ & $2(7)$ & $0(0)$ & 0.2 \\
\hline
\end{tabular}

${ }^{\mathrm{a}}$ The clinical data of the postoperative course are presented as median and range or individual values. AKI, Acute kidney injury; ICU, Intensive care unit; MAP, Mean arterial pressure; p.o., Postoperatively; RRT, Renal replacement therapy; $\mathrm{SaO}_{2}$, Arterial oxygen saturation; $\mathrm{ScvO} \mathrm{O}_{2}$, Systemic venous oxygen saturation. 
Table 4 Intra- and postoperative renal near-infrared spectroscopy scores of infants with versus without acute kidney injury ${ }^{\mathrm{a}}$

\begin{tabular}{|c|c|c|c|}
\hline & AKI & No AKI & $P$-value \\
\hline Renal NIRS <65\% intraoperative (min\%) & 598 (86 to 2,532$)$ & 154 (0 to 2,997$)$ & $0.01^{*}$ \\
\hline Renal NIRS <65\% 12 hr p.o. (min\%) & 694 (0 to 11,534$)$ & $26(0$ to 3,124$)$ & $0.02^{*}$ \\
\hline Renal NIRS <65\% 24 hr p.o. (min\%) & $3,747$ (0 to 22,590$)$ & 267 (1 to 9,417$)$ & $0.007^{*}$ \\
\hline Renal NIRS <65\% 48 hr p.o. (min\%) & $1,113$ (0 to 21,740$)$ & $150(0$ to 8,153$)$ & $0.04^{*}$ \\
\hline Renal NIRS $\triangle 25 \%$ intraoperative (min\%) & $131(0$ to 1,200$)$ & $0(0$ to 2,263$)$ & $0.001^{*}$ \\
\hline Renal NIRS $\triangle 25 \% 12$ hr p.o. (min\%) & $4(0$ to 7,091$)$ & $0(0$ to 1,772$)$ & $0.04^{*}$ \\
\hline Renal NIRS $\triangle 25 \% 24$ hr p.o. (min\%) & $214(0$ to 13,267$)$ & $4(0$ to 312$)$ & $0.02^{*}$ \\
\hline Renal NIRS $\triangle 25 \% 48$ hr p.o. (min\%) & $25(0$ to 6,935$)$ & $0(0$ to 3,518$)$ & 0.1 \\
\hline
\end{tabular}

${ }^{a}$ The renal near-infrared spectroscopy (NIRS) decrease below the baseline of $65 \%$ and the decrease of $25 \%$ below the individualized preoperative renal NIRS intraoperatively and 12, 24 and 48 hours postoperatively are expressed as median and range. AKI, Acute kidney injury; p.o., Postoperatively. ${ }^{*} P<0.05$ indicates statistically significant values.

postoperatively in comparison with patients without AKI (Table 5).

The renal biomarker NGAL in urine and plasma showed a significant increase from the preoperative to the 2 to 4 hours postoperative values (NGAL in urine: 2.4 to $25 \mathrm{ng} / \mathrm{ml}, P>0.0001$; NGAL in plasma: 35 to $41 \mathrm{ng} / \mathrm{ml}, P=0.05)$. NGAL in urine or in plasma did not show a significant difference between patients with and without AKI 2 to 4 hours and 24 hours postoperatively.

\section{Discussion}

The major finding of this study is the significant correlation of an intraoperatively measured renal NIRS score with the postoperative occurrence of AKI in infants after CPB surgery. Second, we found that renal NIRS monitoring performed better than selected biomarkers of renal injury (cystatin $\mathrm{C}$, NGAL) in predicting AKI.

After CPB surgery, AKI in infants with congenital heart disease is a common complication. Its incidence is reportedly between $30 \%$ and $64 \%$ [23,24]. Occurrence of AKI leads to increased morbidity, and several authors have reported increased mortality [3,4]. Various risk factors for the development of AKI have been noted, among them age, bypass time and RACHS-1 score; however, sufficiently sensitive and specific early markers of renal injury have not been consistently defined [1].

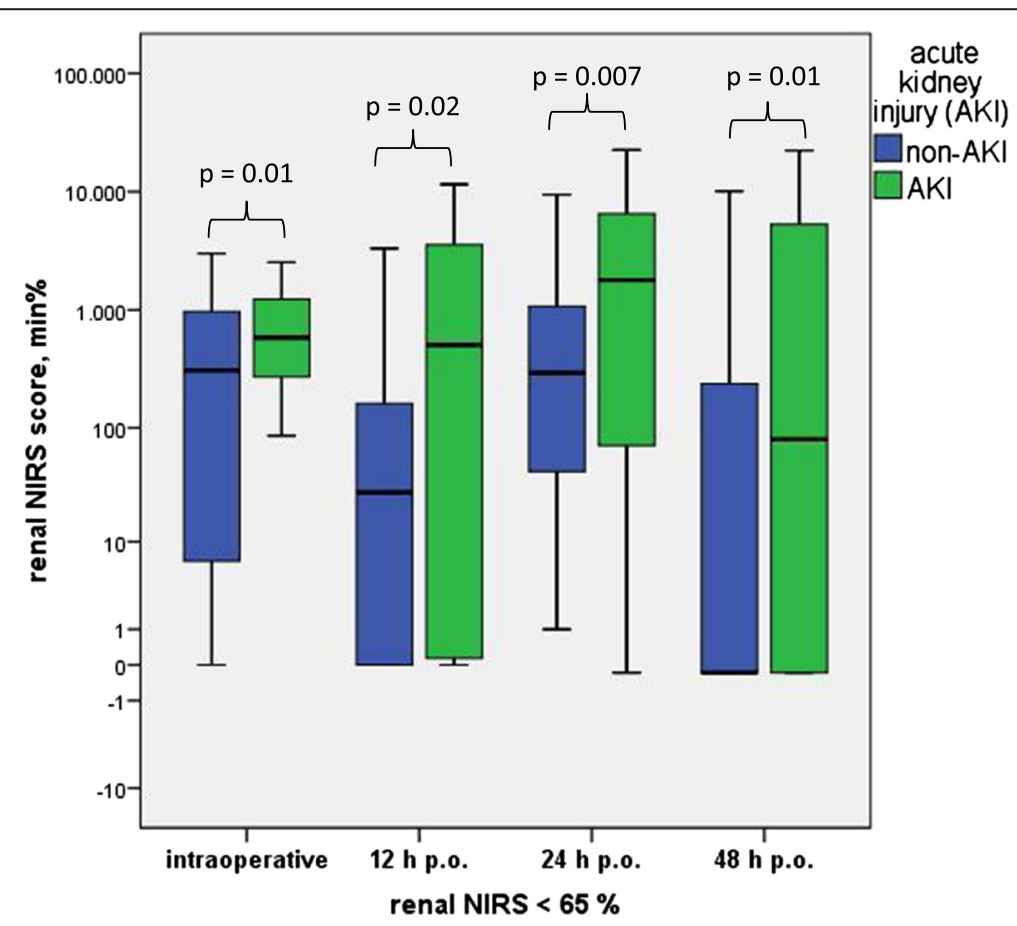

Figure 4 Renal near-infrared spectroscopy score in minute percent intraoperatively and 12, 24 and 48 hours postoperatively below the set baseline of regional oximetry $<65 \%$ in infants with versus without acute kidney injury undergoing cardiopulmonary bypass operations. AKI, Acute kidney injury; NIRS, Near-infrared spectroscopy; p.o., Postoperatively. 


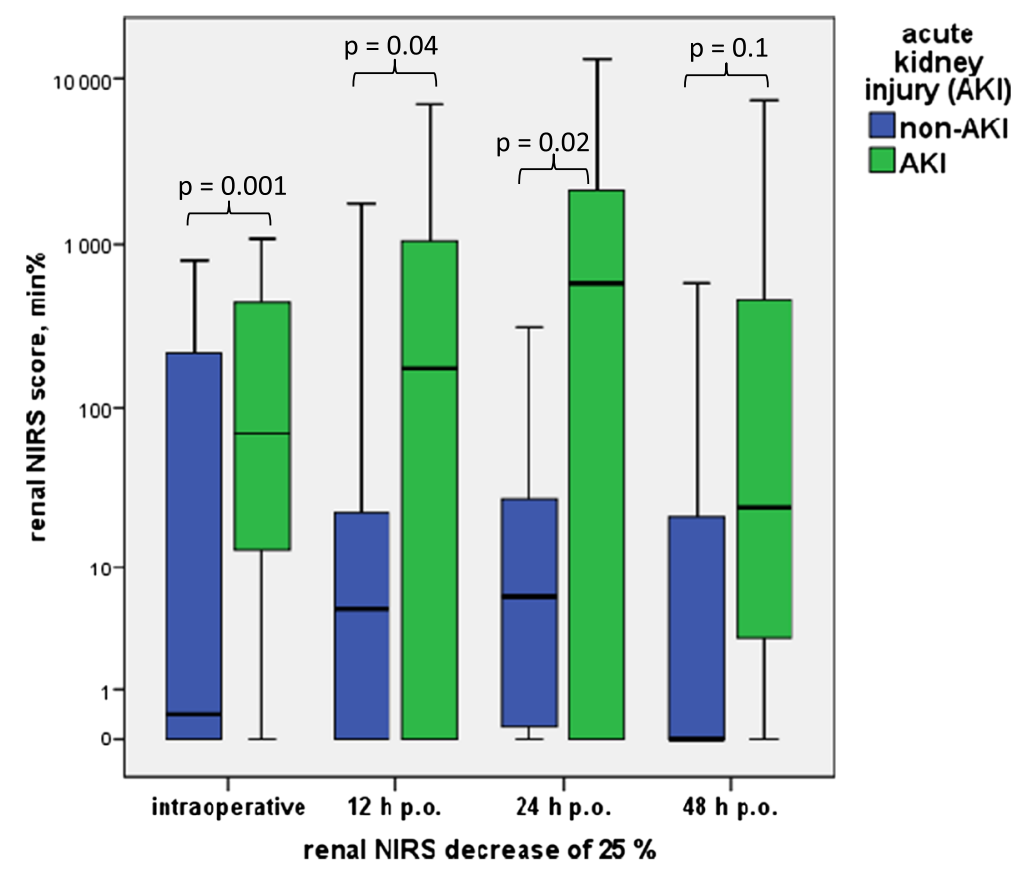

Figure 5 Renal near-infrared spectroscopy score in minute percent intraoperatively and at 12, 24 and 48 hours postoperatively below the set threshold regional oximetry decrease $>\mathbf{2 5} \%$ of the preoperative averaged baseline value in infants with versus without acute kidney injury undergoing cardiopulmonary bypass operations. AKI, Acute kidney injury; NIRS, Near-infrared spectroscopy; p.O., Postoperatively.

Somatic NIRS is thought to reflect tissue perfusion. We postulated that renal $\mathrm{rSO}_{2}$ monitoring might have prognostic value for developing AKI. Renal NIRS could serve as a readily and continuously measurable and, most importantly, early parameter that would allow early intervention trials to modify renal outcomes.

Validated critical baselines for renal $\mathrm{rSO}_{2}$ are lacking and do not discern between cyanotic and acyanotic cardiac lesions. Moreover, the definition of pathological NIRS values is not consistent over studies, ranging from $50 \%$ to $80 \%$, and do not account for the magnitude and duration of desaturations. We set the critical baseline at $65 \% \mathrm{rSO}_{2}$ for renal NIRS according to the threshold of $45 \%$ to $55 \%$ accepted in cerebral NIRS studies [17-19] and the 10 to 20 points higher somatic than cerebral values in healthy infants [20,21]. To account for a priori

Table 5 Pre- and postoperative cystatin C levels of infants with versus without acute kidney injury ${ }^{\mathrm{a}}$

\begin{tabular}{llll}
\hline & AKI & No AKI & $P$-value \\
\hline Cystatin C, mg/L & $1.4(0.7$ to 1.9$)$ & $1.4(0.6$ to 2.8$)$ & 0.87 \\
Preoperatively & $1.1(0.6$ to 1.3$)$ & $1.1(0.7$ to 1.2$)$ & 0.96 \\
Postoperatively & $1.7(1.0$ to 2.7$)$ & $1.4(0.8$ to 2.2$)$ & 0.002 \\
24 hours postoperatively & $1.7(1.0$ to 2.1$)$ & $1.4(0.8$ to 2.6$)$ & 0.02 \\
\hline 48 hours postoperatively & 1.02 &
\end{tabular}

${ }^{\mathrm{a}}$ The renal biomarker serum cystatin $\mathrm{C}$ is presented pre- and postoperatively and 24 and 48 hours after surgery as median and range. AKI, Acute kidney injury. lower NIRS values in patients with cyanotic lesions, we defined a second criterion, which includes relative decreases in regional oxygenation compared with an individually calculated baseline. We speculated that, as baseline values are already low in cyanotic patients, a parameter of a relative decrease in saturation values might be more sensitive in the detection of a perfusion compromise. In addition, the area under the threshold lines was calculated as NIRS score to account for magnitude and duration of desaturations.

In two somatic NIRS studies, it has already been shown that lower renal $\mathrm{rSO}_{2}$ after surgery correlates with occurrence of AKI in infants after CPB $[20,25]$. Only one of these studies [20] included NIRS measurements intraoperatively, but no correlation with AKI was reported. In each of these studies, different cutoff values for renal $\mathrm{rSO}_{2}$ were used to define critical desaturation episodes, including criteria for relevant time periods of desaturations, which impedes comparisons between studies. In contrast to these studies, we could show a significant difference in renal desaturation, by our criteria, between infants with and those without AKI developing at the earliest time point (that is, during surgery). As $\mathrm{CPB}$ alters oxygen delivery, this difference was most distinct after stopping CPB; however, it was consistent over 12 and 24 hours postoperatively.

Our results show that both NIRS criteria correlate with renal outcome and haemodynamic parameters and surprisingly did not differ significantly in patients with 
and without cyanosis, independent of the development of AKI. Efficient mechanisms seem to be functional in cyanotic patients to compensate for lower systemic arterial oxygen saturation. Therefore, we propose that, although systemic arterial oxygen saturations are lower in cyanotic patients and the majority of AKI patients were cyanotic, systemic cyanosis seems not to be the cause of the significant difference in renal NIRS values between AKI and non-AKI groups.

In various clinical studies in neonates and infants, somatic NIRS has been used as an indicator of tissue perfusion and has been established as a monitoring tool in the haemodynamic management of critically ill infants $[11,13,25]$. Several studies have demonstrated a moderate correlation of somatic $\mathrm{rSO}_{2}$ with lactate and central venous saturation as indicators of cardiac output [12,26,27]. Uncertainty remains, however, because other studies have failed to identify a correlation between regional NIRS and several cardiac output parameters [28,29]. Abdominal site NIRS [14] or a combination of cerebral and renal NIRS [27] may perform best in this regard. With more specific regard to the kidneys, a good correlation of renal vein and inferior vena cava saturation with renal NIRS in children weighing $<10 \mathrm{~kg}$ has been demonstrated [30]. Our results support the notion that somatic NIRS is dependent on cardiac output and reflects tissue perfusion, as we could show a correlation, although it was moderate between renal NIRS scores intraoperatively and systemic lactate levels 6 and 24 hours after surgery. Further, renal NIRS scores in the first 24 hours after surgery correlated significantly with $\mathrm{ScvO}_{2}$ at 2 and 24 hours, as well as with systemic lactate levels at 24 hours after surgery.

In our study population, $48 \%$ of infants developed AKI after CPB surgery, which is comparable to recently published series [1,5]. Known risk factors for the development of AKI are younger age [1,3-5,31], bypass time [1-3,5], RACHS-1 score [3,5] and vasopressor use [2]. Infants, especially newborns, seem to be at highest risk for developing AKI after CPB surgery [1,5]. In our study, patients in the AKI group were significantly younger than patients in the non-AKI group, confirming these findings, whereas there was no difference in RACHS-1 scores.

$\mathrm{CPB}$ per se and haemodynamic compromise during the intra- and postoperative course could be responsible for postoperative AKI [32]. In our cohort, CPB time showed no significant difference between infants who developed AKI and infants who did not. Lactate after 24 hours in infants with AKI is significantly higher and $\mathrm{ScvO}_{2}$ after 24 hours in infants with AKI is significantly lower than in infants without AKI. In addition, we noted lower blood pressures and higher vasopressor requirements in the patients with AKI. Last, the number of patients with systemicopulmonary shunts was significantly higher in the AKI group. Haemodynamic conditions in systemicopulmonary shunt palliation can influence renal perfusion because of diastolic run-off and lower diastolic blood pressure [33]. In summarizing these findings, in our study population, peri- and postoperative haemodynamic compromise was a major factor in the development of postoperative AKI. Importantly, intraoperative renal NIRS reflected these pathologies at the earliest time point.

Over the last several years, renal biomarkers for use in predicting the development of AKI earlier than serum creatinine have been investigated extensively, but firm conclusions have not been reached [8]. In our present study, we confirmed earlier findings [34] that plasma cystatin $C$ increased significantly after CPB surgery in patients with postoperative AKI, but showed a significant increase only at least 24 hours postoperatively, whereas others have noted an earlier rise [35].

With regard to serum and urinary NGAL, we did not find a significant difference between infants who developed AKI and those who did not. This finding is in contrast to several earlier reports [36-38] of impressive AUROC values, although moderate AUROC values have been noted as well $[9,39]$. Moreover, the optimal time point of NGAL measurements and optimal cutoff values have not been defined. We noted that in earlier studies [36-39], CPB time differed significantly between AKI and non-AKI groups, which was not the case in our study. Therefore, NGAL values may have a close correlation with $\mathrm{CPB}$ time and a less direct correlation with AKI incidence. In our study, neither NGAL nor cystatin C proved useful for early diagnosis of AKI after CPB. As other authors have suggested, a multimodal prognostic model that incorporates several biomarkers, clinical risk factors and NIRS values may serve best in predicting AKI $[9,16]$.

A limitation of our study is the use of a newly defined analysis of NIRS values in infants, which has to be validated in further studies. Furthermore, we did not perform either (1) a continuous measurement of central venous saturation as an indirect real-time marker for cardiac output and tissue perfusion or (2) a thermodilution measurement as a direct real-time marker for cardiac output and tissue perfusion.

In summary, we found that continuous renal NIRS monitoring, starting intraoperatively and extending postoperatively, in infants after cardiac surgery may be a valuable and, importantly, very early parameter for predicting AKI. Of great practical value are its non-invasive and continuous character and the potential to deliver instantaneous values. We developed two methods of analysis, including absolute and relative cutoff values. rNIRS scores $<65 \%$ show a high sensitivity, and relative rNIRS values $<25 \%$ have acceptable specificity, both of which may be improved by refining thresholds in larger patient cohorts. In our series, intraoperative renal NIRS was superior to conventional biomarkers of renal injury for 
early detection of developing AKI, although a combined model that incorporates biomarkers and renal NIRS may be expected to have increased sensitivity and specificity.

\section{Conclusion}

Renal NIRS, when performed during and after infant cardiac surgery, is a promising tool to detect early haemodynamic compromise and predict development of AKI. Renal NIRS monitoring therefore should be included in prognostic models for early identification of renal injury risk in infants during and after $\mathrm{CPB}$ operation and may allow the development of therapeutic strategies to avoid kidney injury during cardiac surgery in infants.

\section{Key message}

- Renal regional NIRS, when started intraoperatively and with specific thresholds, allows very early risk detection of the development of AKI after CPB surgery in infants.

\begin{abstract}
Abbreviations
AKI: Acute kidney injury; CPB: Cardiopulmonary bypass; ICU: Intensive care unit; NGAL: Neutrophil gelatinase-associated lipocalin; NIRS: Near-infrared spectroscopy; NPV: Negative predictive value; PPV: Positive predictive value; pRIFLE: Paediatric Risk, Injury, Failure, Loss, End; RACHS-1: Risk Adjustment for Congenital Heart Surgery; $\mathrm{rSO}_{2}$ : Regional oximetry; RRT: Renal replacement therapy; $\mathrm{ScvO}_{2}$ : Venous oxygen saturation.
\end{abstract}

\section{Competing interests}

The authors declare that they have no competing interests.

\begin{abstract}
Authors' contributions
BR made substantial contributions to study conception and design and the acquisition, analysis and interpretation of data and drafted the manuscript. VB made substantial contributions to the acquisition of data. GB participated in the design of the study and revised the manuscript critically. JH and NN were involved in revising the manuscript critically. SLB carried out the laboratory analysis and drafted the laboratory analysis section of the manuscript. PE revised the manuscript critically. KR made substantial contributions to study conception and design and participated in writing the manuscript. All authors read and approved the final manuscript.
\end{abstract}

\section{Author details \\ 'Department of Pediatric Cardiology and Congenital Heart Disease, German Heart Centre Munich, Technical University, Lazarettstr. 36, 80636 Munich, Germany. ${ }^{2}$ Department of Anaesthesiology, German Heart Centre Munich, Technical University, Lazarettstr. 36, 80636 Munich, Germany. ${ }^{3}$ Department of Thoracic and Cardiovascular Surgery, German Heart Centre Munich, Technical University, Lazarettstr. 36, 80636 Munich, Germany. ${ }^{4}$ Institute of Laboratory Medicine, German Heart Centre Munich, Technical University, Lazarettstr. 36, 80636 Munich, Germany. ${ }^{5}$ Department of Pediatric Intensive Care, University Children`s Hospital, von Haunersche Children hospital, Ludwigs-Maximilian University, Lindwurmstr. 4, 80337 Munich, Germany.}

Received: 24 March 2014 Accepted: 20 January 2015

Published online: 29 January 2015

\section{References}

1. Li S, Krawczeski CD, Zappitelli M, Devarajan P, Thiessen-Philbrook H, Coca SG, et al. Incidence, risk factors, and outcomes of acute kidney injury after pediatric cardiac surgery: a prospective multicenter study. Crit Care Med. 2011;39:1493-9.

2. Zappitelli M, Bernier PL, Saczkowski RS, Tchervenkov Cl, Gottesman R, Dancea A, et al. A small post-operative rise in serum creatinine predicts acute kidney injury in children undergoing cardiac surgery. Kidney Int. 2009;76:885-92.

3. Pedersen K. Acute kidney injury in children undergoing surgery for congenital heart disease. Eur J Pediatr Surg. 2012;22:426-33.

4. Blinder JJ, Goldstein SL, Lee W, Baycroft A, Fraser CD, Nelson D, et al. Congenital heart surgery in infants: effects of acute kidney injury on outcomes. J Thorac Cardiovasc Surg. 2012;143:368-74.

5. Aydin SI, Seiden HS, Blaufox AD, Parnell VA, Choudhury T, Punnoose A, et al. Acute kidney injury after surgery for congenital heart disease. Ann Thorac Surg. 2012;94:1589-95.

6. Akcan-Arikan A, Zappitelli M, Loftis LL, Washburn KK, Jefferson LS, Goldstein SL. Modified RIFLE criteria in critically ill children with acute kidney injury. Kidney Int. 2007;71:1028-35.

7. Devarajan P. Biomarkers for the early detection of acute kidney injury. Curr Opin Pediatr. 2011;23:194-200.

8. Vanmassenhove J, Vanholder R, Nagler E, Van Biesen W. Urinary and serum biomarkers for the diagnosis of acute kidney injury: an in-depth review of the literature. Nephrol Dial Transplant. 2013;28:254-73.

9. Alcaraz AJ, Gil-Ruiz MA, Castillo A, López J, Romero C, Fernández SN, et al. Postoperative neutrophil gelatinase-associated lipocalin predicts acute kidney injury after pediatric cardiac surgery. Pediatr Crit Care Med. 2014;15:121-30.

10. Murkin JM, Arango M. Near-infrared spectroscopy as an index of brain and tissue oxygenation. Br J Anaesth. 2009;103 Suppl 1:13-13.

11. Hoffmann GM, Stuth EA, Jaquiss RD, Vanderwal PL, Staudt SR, Troshynski TJ, et al. Changes in cerebral and somatic oxygenation during stage 1 palliation of hypoplastic left heart syndrome using continuous regional cerebral perfusion. J Thorac Cardiovasc Surg. 2004;127:223-33.

12. Ranucci M, Isgrò G, De la Torre T, Romitti F, Conti D, Carlucci C. Near-infrared spectroscopy correlates with continuous superior vena cava oxygen saturation in pediatric cardiac surgery patients. Pediatr Anesth. 2008:18:1163-9.

13. Hanson SJ, Berens RJ, Havens PL, Kim MK, Hoffmann GM. Effect of volume resuscitation on regional perfusion in dehydrated pediatric patients as measured by two- site near-infrared spectroscopy. Pediatr Emerg Care. 2009;25:150-3.

14. Kaufman J, Almodovar MC, Zuk J, Friesen RH. Correlation of abdominal site near-infrared spectroscopy with gastric tonometry in infants following surgery for congenital heart disease. Pediatr Crit Care Med. 2008;9:62-8.

15. Bhalala U, Nishisaki A, McQueen D, Bird GL, Morrison WE, Nadkarni VM, et al. Change in regional (somatic) near-infrared spectroscopy is not a useful indictor of clinically detectable low cardiac output in children after surgery for congenital heart defects. Pediatr Crit Care Med. 2012;13:529-34.

16. Hazle MA, Gajarski RJ, Aiyagari R, Yu S, Abraham A, Donohue J, et al. Urinary biomarkers and renal near-infrared spectroscopy predict intensive care unit outcomes after cardiac surgery in infants younger than 6 months of age. J Thorac Cardiovasc Surg. 2013;146:861-7.

17. Slater JP, Guarino T, Stack J, Vinod K, Bustami RT, Brown 3rd JM, et al. Cerebral oxygen desaturation predicts cognitive decline and longer hospital stay after cardiac surgery. Ann Thorac Surg. 2009;87:36-45.

18. Dent CL, Spaeth JP, Jones BV, Schwartz SM, Glauser TA, Hallinan B, et al. Brain magnetic resonance imaging abnormalities after the Norwood procedure using regional cerebral perfusion. J Thorac Cardiovasc Surg. 2005;130:1523-30

19. Kussman BD, Wypij D, Laussen PC, Soul JS, Bellinger DC, DiNardo JA, et al. Relationship of intraoperative cerebral oxygen saturation to neurodevelopmental outcome and brain magnetic resonance imaging at 1 year of age in infants undergoing biventricular repair. Circulation. 2010;122:245-54.

20. Colasacco C, Worthen M, Peterson B, Lamberti J, Spear R. Near-infrared spectroscopy monitoring to predict postoperative renal insufficiency following repair of congenital heart disease. World J Pediatr Cong Heart Surg. 2011;2:536-40.

21. Bernal NP, Hoffmann GM, Ghanayem NS, Arca MJ. Cerebral and somatic nearinfrared spectroscopy in normal newborns. J Pediatr Surg. 2010;45:1306-10.

22. Jenkins KJ, Gauvreau K, Newburger JW, Spray TL, Moller JH, lezzoni LI. Consensus-based method for risk adjustment for surgery for congenital heart disease. J Thorac Cardiovasc Surg. 2002;123:110-8.

23. Krawczeski CD, Woo JG, Wang Y, Bennet MR, Ma Q, Devarajan P. Neutrophil gelatinase-associated lipocalin concentrations predict development of acute kidney injury in neonates and children after cardiopulmonary bypass. J Pediatr. 2011;158:1009-15.e1. 
24. Morgan CJ, Zappitelli M, Robertson CMT, Alton GY, Sauve RS, Joffe AR, et al. Risk factors for and outcomes of acute kidney injury in neonates undergoing complex cardiac surgery. J Pediatr. 2013;162:120-127.e1.

25. Owens GE, King K, Gurney JG, Charpie JR. Low renal oximetry correlates with acute kidney injury after infant cardiac surgery. Pediatr Cardiol. 2011:32:183-8.

26. Nagdyman N, Ewert P, Peters B, Miera O, Fleck T, Berger F. Comparison of different near-infrared spectroscopic cerebral oxygenation indices with central venous and jugular venous oxygenation saturation in children. Paediatr Anaesth. 2008;18:160-6.

27. Chakravati SB, Mittnacht AJ, Katz JC, Nguyen K, Joashi U, Svrivastava S. Multisite near-infrared spectroscopy predicts elevated blood lactate level in children after cardiac surgery. J Cardiothorac Vasc Anesth. 2009;23:663-7.

28. Moreno GE, Pilán ML, Manara C, Magliola R, Vassallo JC, Balestrini M, et al. Regional venous oxygen saturation versus mixed venous saturation after paediatric cardiac surgery. Acta Anaesthesiol Scand. 2013;57:373-9.

29. McQuillen PS, Nishimoto MS, Bottrell CL, Fineman LD, Hamrick SE, Glidden DV, et al. Regional and central venous oxygen saturation monitoring following pediatric cardiac surgery: concordance and association with clinical variables. Pediatr Crit Care Med. 2007;8:154-60.

30. Ortmann LA, Fontenot EE, Seib PM, Eble BK, Brown R, Bhutta AT. Use of near-infrared spectroscopy for estimation of renal oxygenation in children with heart disease. Pediatr Cardiol. 2011;32:748-53.

31. Zappitelli M. Preoperative predictors of acute kidney injury — from clinical scores to biomarkers. Pediatr Nephrol. 2013;28:1173-82.

32. Rosner MH, Okusa MD. Acute kidney injury associated with cardiac surgery Clin J Am Soc Nephrol. 2006;1:19-32.

33. Malec E, Januszewska K, Kolcz J, Mroczek T. Right ventricle-to-pulmonary artery shunt versus modified Blalock-Taussig shunt in the Norwood procedure for hypoplastic left heart syndrome - influence on early and late haemodynamic status. Eur J Cardiothorac Surg. 2003;23:728-34.

34. Zappitelli M, Krawczeski CD, Devarajan P, Wang Z, Sint K, Thiessen-Philbrook H, et al. Early postoperative serum cystatin C predicts severe acute kidney injury following pediatric cardiac surgery. Kidney Int. 2011;80:655-62.

35. Krawczeski CD, Vandervoorde RG, Kathman T, Bennett MR, Woo JG, Wang Y, et al. Serum cystatin $C$ is an early predictive biomarker of acute kidney injury after pediatric cardiopulmonary bypass. Clin J Am Soc Nephrol. 2010:5:1552-7.

36. Mishra J, Dent C, Tarabishi R, Mitsnefes MM, Ma Q, Kelly C, et al. Neutrophil gelatinase-associated lipocalin (NGAL) as a biomarker for acute renal injury after cardiac surgery. Lancet. 2005;365:1231-8.

37. Dent $C L$, Ma Q, Dastrala S, Bennet M, Mitsnefes MM, Barasch J, et al. Plasma neutrophil gelatinase-associated lipocalin predicts acute kidney injury, morbidity and mortality after pediatric cardiac surgery: a prospective uncontrolled cohort study. Crit Care. 2007;11:R127.

38. Bennet M, Dent CL, Ma Q, Dastrala S, Grenier F, Workman R, et al. Urine NGAL predicts severity of acute kidney injury after cardiac surgery: a prospective study. Clin J Am Soc Nephrol. 2008:3:665-73.

39. Parikh CR, Devarajan P, Zappitelli M, Sint K, Thiessen-Philbrook H, Li S, et al. Postoperative biomarkers predict acute kidney injury and poor outcomes after pediatric cardiac surgery. J Am Soc Nephrol. 2011;22:1737-47.

\section{Submit your next manuscript to BioMed Central and take full advantage of:}

- Convenient online submission

- Thorough peer review

- No space constraints or color figure charges

- Immediate publication on acceptance

- Inclusion in PubMed, CAS, Scopus and Google Scholar

- Research which is freely available for redistribution 